\title{
OPEN Neighbors-based prediction of physical function after total knee arthroplasty
}

\author{
Chong Kim ${ }^{1}$, Kathryn L. Colborn ${ }^{1}$, Stef van Buuren ${ }^{2,3}$, Timothy Loar ${ }^{4}$, \\ Jennifer E. Stevens-Lapsley ${ }^{4,5}$ \& Andrew J. Kittelson ${ }^{4,6} \bowtie$
}

The purpose of this study was to develop and test personalized predictions for functional recovery after Total Knee Arthroplasty (TKA) surgery, using a novel neighbors-based prediction approach. We used data from 397 patients with TKA to develop the prediction methodology and then tested the predictions in a temporally distinct sample of 202 patients. The Timed Up and Go (TUG) Test was used to assess physical function. Neighbors-based predictions were generated by estimating an index patient's prognosis from the observed recovery data of previous similar patients (a.k.a., the index patient's "matches"). Matches were determined by an adaptation of predictive mean matching. Matching characteristics included preoperative TUG time, age, sex and Body Mass Index. The optimal number of matches was determined to be $m=35$, based on low bias ( -0.005 standard deviations), accurate coverage ( $50 \%$ of the realized observations within the $50 \%$ prediction interval), and acceptable precision (the average width of the $50 \%$ prediction interval was $2.33 \mathrm{~s}$ ). Predictions were well-calibrated in out-of-sample testing. These predictions have the potential to inform care decisions both prior to and following TKA surgery.

Total Knee Arthroplasty (TKA) is the most commonly performed inpatient elective surgery in the United States, at approximately 700,000 procedures per year ${ }^{1}$. Although TKA is regarded as effective, the clinical course is highly variable ${ }^{2}$. Depending on the patient, recovery of physical function can occur within weeks, or it can be an arduous months-long process ${ }^{3,4}$. Moreover, the surgical population is remarkably heterogeneous. Some patients engage in sporting activities (e.g., tennis, skiing) ${ }^{5}$, while others struggle to ambulate at walking speeds sufficient for independence in the community. There is no such thing as the "average" patient ${ }^{6}$.

To achieve the ideals of person-centered care ${ }^{7-9}$, and also because TKA is an elective procedure, clinical decisions should be anchored to the individual patient ${ }^{10}$. Yet the determination of an individual patient's functional prognosis is challenging. Prediction models have been developed in TKA, but these models have several limitations: (1) they perform poorly in out-of-sample testing ${ }^{11}$, (2) they are based on mathematical functions that are unlikely to be flexible enough to realistically portray the clinical course across all patients ${ }^{12}$, or (3) they predict functional outcomes at discrete postoperative time points, which may not overlap with the time frame during which patients are undergoing postoperative care and clinical monitoring ${ }^{13}$.

Neighbors-based predictions may overcome some of these limitations. In a neighbors-based approach, an index patient's prognosis is estimated from the observed recovery data of previous similar patients ${ }^{14}$. These previous patients are known as the index patient's neighbors or "matches". In this approach, the parameters of the prediction and the shape of the prognostic trajectory are allowed to vary substantially across individuals. Such flexibility may better accommodate the heterogeneity in recovery following TKA ${ }^{15}$. This may also enhance the generalizability of the approach. The prediction is generated based only on a subset of patients with characteristics similar to the index patient; this contrasts with traditional prediction approaches where model parameters are heavily informed by the aggregated characteristics of the sample.

The purpose of this study was to develop and test a neighbors-based prediction approach for functional recovery after TKA surgery ${ }^{16}$. The outcome of interest for this analysis was the Timed Up and Go (TUG) test, a

\footnotetext{
${ }^{1}$ Department of Biostatistics and Informatics, Colorado School of Public Health, University of Colorado, Aurora, CO, USA. ${ }^{2}$ Netherlands Organization for Applied Scientific Research TNO, Leiden, The Netherlands. ${ }^{3}$ Department of Methodology and Statistics, University of Utrecht, Utrecht, The Netherlands. ${ }^{4}$ Physical Therapy Program, Department of Physical Medicine and Rehabilitation, University of Colorado, Aurora, CO, USA. ${ }^{5}$ Eastern Colorado VA Geriatric Research, Education, and Clinical Center (GRECC), VA Eastern Colorado Health Care System, Aurora, CO, USA. ${ }^{6}$ School of Physical Therapy and Rehabilitation Science, University of Montana, Missoula, MT, USA. ${ }^{\circledR}$ email: Andy.Kittelson@umontana.edu
} 


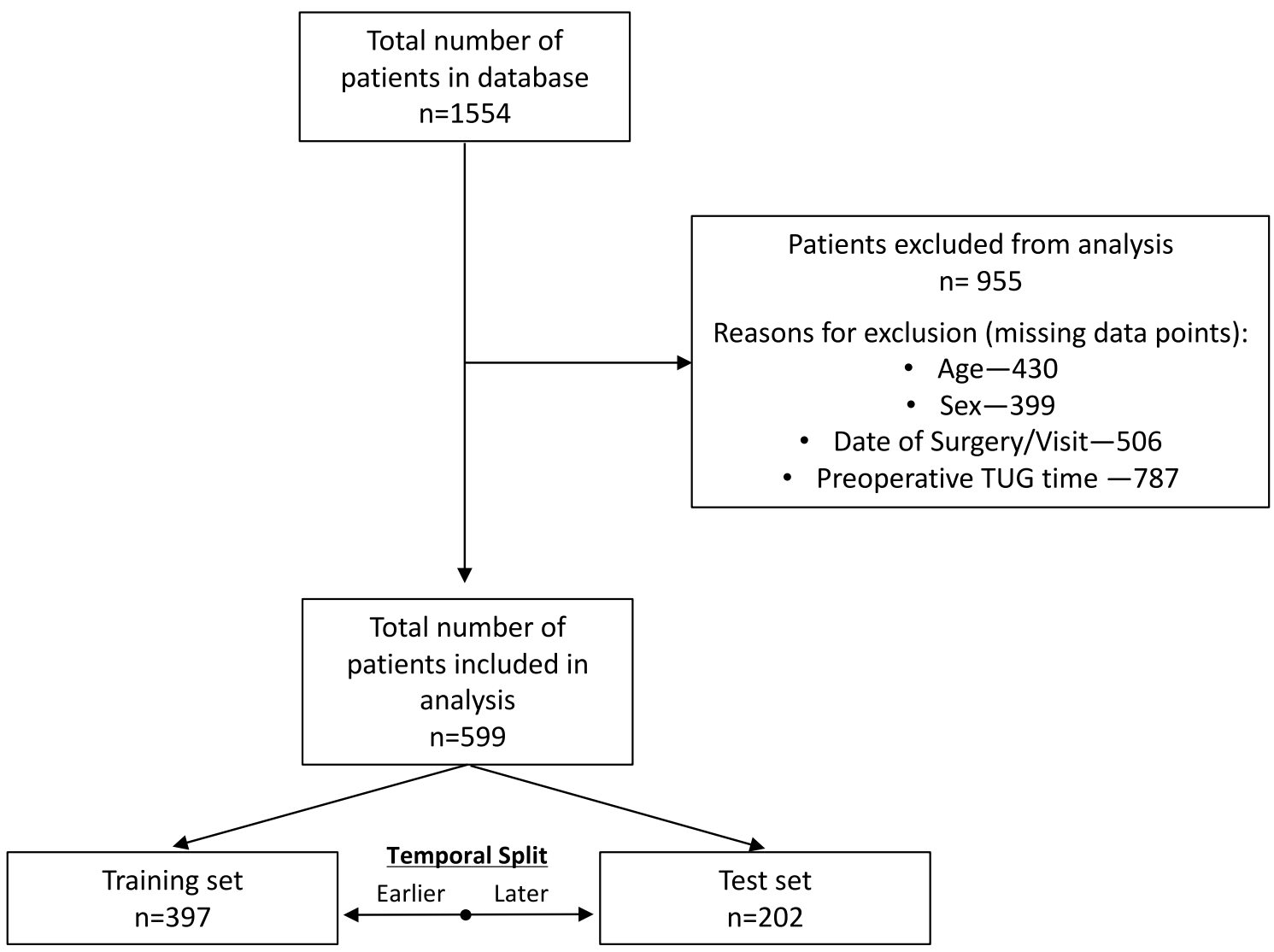

Figure 1. CONSORT flow diagram of patient data included in the analysis.

clinically feasible test of mobility and a surrogate of lower extremity strength. We utilized a combination of clinical and research data, collected longitudinally over the first six months following surgery. We divided the patients temporally (by date of surgery) into a training set and test set. This split was made to mimic how the approach would be developed and tested in clinical practice. The training set was used to tune the neighbors-based prediction, particularly to choose the optimal number of matches required to achieve optimal performance. The training set then also served as the donor dataset for an out-of-sample validation using patients from the test set.

\section{Methods}

Data sources. This analysis utilized two existing data sources involving patients with primary, unilateral TKA: (1) data collected in routine clinical practice and (2) data from previously published longitudinal research studies. Clinical data were obtained via routine quality improvement procedures at ATI physical therapy (Greenville, SC), with surgery dates between January, 2013 and June, 2017. Research data were obtained from four previously published studies, with surgery dates between June, 2006 and May, 2017. The inclusion/exclusion criteria for these research studies have been reported elsewhere ${ }^{17-20}$. Clinical data were not selected based on patient criteria (i.e., all patients with clinical visits were included in the dataset), although only patient records containing a preoperative and postoperative TUG assessment were utilized in this analysis. The combined dataset was divided temporally, based on surgical date, into a training set and a test set (Fig. 1). All participants provided informed consent. All records were de-identified prior to use in this study, and all methods were approved by the Colorado Multiple Institutional Review Board (COMIRB) and carried out in accordance with relevant regulations.

Timed up and go (TUG) test. The TUG is a brief test of mobility, where a patient rises from a chair, walks a distance of $3 \mathrm{~m}$ and returns to a seated position in the chair. Patients were instructed to perform the test, "as quickly but as safely as possible". The TUG demonstrates high test-retest reliability and responsiveness ${ }^{12,21,22}$. All testers involved with data collection for this analysis followed the same set of standardized instructions for performing the TUG test ${ }^{22}$.

Matching characteristics. Variables used for selecting matches were patient factors common across all datasets: age (years), sex, Body Mass Index (BMI; $\mathrm{kg} / \mathrm{m}^{2}$ ), and preoperative TUG time (seconds).

Statistical analysis. All analyses were conducted using R version 3.5.1. The steps to generate a neighborsbased prediction by predictive mean matching are summarized in the following sections and also described in Supplementary Material (Box 1). 


\begin{tabular}{|l|l|l|l|}
\hline & Train & Test & \multirow{2}{*}{ p-value ${ }^{\boldsymbol{a}}$} \\
\cline { 2 - 3 } & $\mathbf{( n = 3 9 7 , 1 3 3 9}$ observations) & $(\mathbf{n}=\mathbf{2 0 2}, \mathbf{6 0 4}$ observations $)$ & 0.012 \\
\hline Age, years; mean (sd) & $64.04(8.43)$ & $65.90(8.84)$ & 0.280 \\
\hline Sex distribution, n (\% male) & $185(46.6)$ & $84(41.6)$ & 0.208 \\
\hline BMI, kg/m²; mean (sd) & $31.33(5.82)$ & $31.98(6.20)$ & 0.018 \\
\hline Preop TUG, seconds; mean $(\mathrm{sd})$ & $9.98(4.95)$ & $11.00(5.04)$ & \\
\hline
\end{tabular}

Table 1. Baseline characteristics of training and test datasets. ${ }^{a}$ Continuous variables tested with one-way analysis of variance; Categorical variables tested with $\chi^{2}$ test. Preop TUG, preoperative timed up and go time; sd, standard deviation; BMI, body mass index.

Selection of matches by predictive mean matching. Because the source datasets contained TUG assessments at irregular postoperative time-points, we estimated a 90-day postoperative TUG time for all patients using linear mixed effects models via the brokenstick package ( $\mathrm{R}$ statistical computing etc. ${ }^{23-25}$. The 90-day time-point was used as the distal anchor for selecting matches by predictive mean matching ${ }^{26}$. Briefly, a brokenstick model was fit to patients in the training data with 4 knots at specific timepoints after surgery $(\mathrm{k}=0$; $14 ; 50 ; 90)$. Patients in the training data were then matched according to the 90 -day predicted TUG time by building a linear model with matching characteristics as predictors and the 90 -day brokenstick-estimated TUG time as the outcome variable.

Flexible modeling of observed data. For each patient in the training data, the observed postoperative TUG data of the patient's matches were used to fit a Generalized Additive Model for Location Scale and Shape (GAMLSS) $^{27}$. The GAMLSS model was chosen for its flexibility in modeling the median (location), variance (scale), skewness and kurtosis (shape) of the TUG as a smooth function of time (i.e., time since TKA). In particular, since TUG times are positively skewed it was preferable to employ a modeling framework that accommodates flexibility in skewness over time. A cubic spline smoother with 3 degrees of freedom (df) for the location parameter and $1 \mathrm{df}$ for the scale and shape parameters was employed.

Model tuning via within-sample testing. The optimal number of matches $(\mathrm{m})$ was chosen by the following procedure: (1) GAMLSS models were fit to the matches' observed data for each of the 397 patients in the training set, with the number of matches ranging from 10 to 397 (i.e., the total number of available patients in the training data), (2) at each increment (i.e., 10 matches; 11; 12; : : ; 397 matches), the average bias, coverage, and precision of the predictions were calculated, and (3) the optimal number of matches was determined globally by the solution that minimized bias and optimized precision whilst retaining accurate coverage (see Supplementary Material; Box 2).

Internal and external validation. To test the performance of the predictions, we compared predicted vs. observed TUG times via calibration plots. For both the training and test sets, we binned the predicted TUG times by deciles. Within each decile of predicted data, the median and the standard error (95\% Confidence Interval) of the observed data were calculated. The median was a better measure of central tendency given the skewness of the TUG data.

\section{Results}

In the training data set we analyzed information on 397 patients with 1,339 post-operative TUG observations. We used information on 202 patients (604 observations) in the testing data. Patient characteristics from training and testing data are shown in Table 1 . Although the sex distribution and BMI were similar across the two data sets, there were statistically significant differences in age and baseline TUG time. Compared to the patients in the training data, patients in the test data were approximately 2 years older on average, with $1 \mathrm{~s}$ slower baseline TUG times.

Selection of matches and model tuning. Predictive mean matching. Age $(\beta=0.037 ; \mathrm{p}=0.001)$, sex $(B=0.92 ; p<0.001)$, BMI $(B=0.037 ; p=0.02)$, and preoperative TUG time $(B=0.21 ; p<0.001)$ demonstrated a statistically significant relationship with brokenstick estimates of the 90 -day post-operative TUG time. Preoperative TUG time carried the biggest weight in selecting matches; the standardized coefficient for preoperative TUG time was 4.7 times larger than for BMI.

Examining the optimal number matches. The optimal number of matches was found to be $\mathrm{m}=35$ based on the low bias ( 0.005 standard deviations) and accurate coverage (proportion of realized observations within the $50 \%$ prediction interval: 0.50 ). Additionally, the average width of the $50 \%$ prediction interval with $\mathrm{m}=35$ matches was $2.33 \mathrm{~s}$ (Fig. 2). With $\mathrm{m}=397$ matches (i.e., the full training dataset), the average precision was $3.03 \mathrm{~s}$. Thus, the neighbors-based prediction with $\mathrm{m}=35$ matches resulted in a $23 \%$ improvement in precision (Fig. 3 ). 

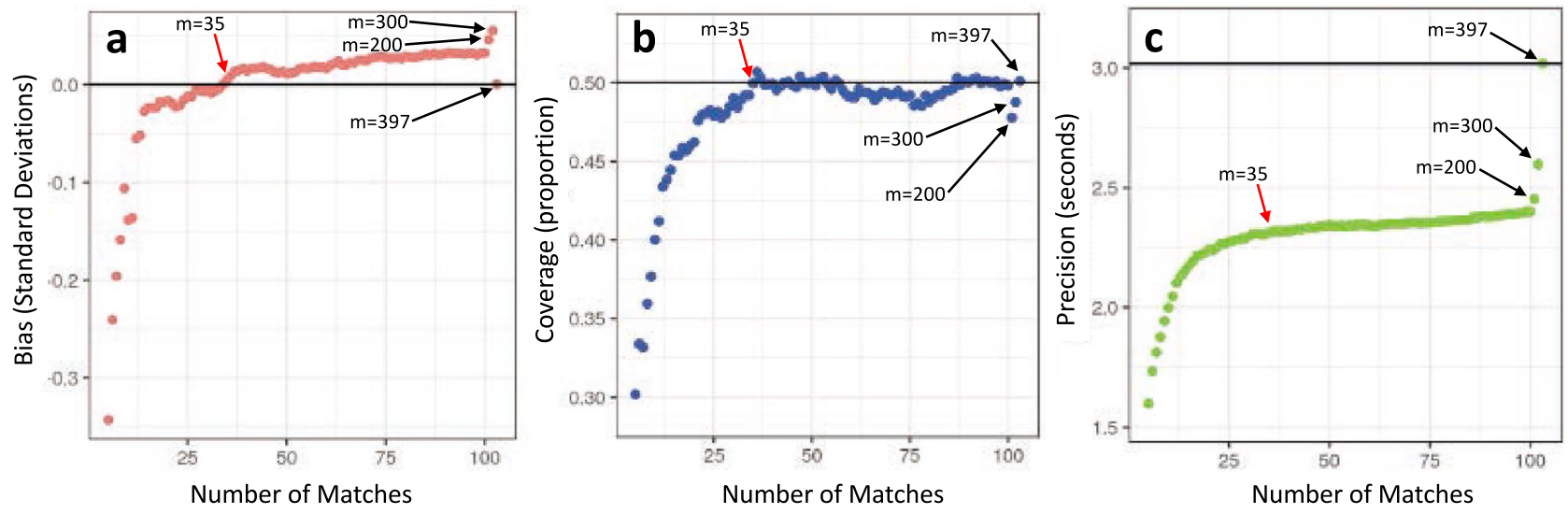

Figure 2. Performance metrics for neighbors-based predictions across increasing number of matches in the training dataset: (a) bias, (b) coverage, and (c) precision. The optimal number of matches $(\mathrm{m}=35)$ is indicated with a red arrow.

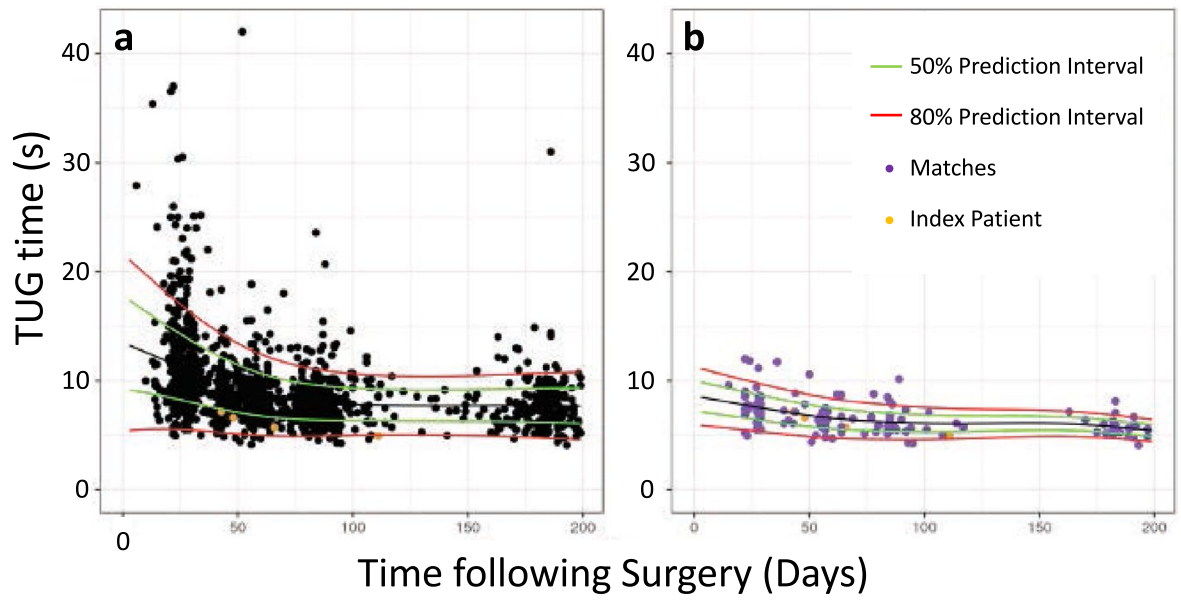

Figure 3. The $50 \%$ prediction interval (PI) for (a) the population-level estimate, is wider than the $50 \%$ prediction interval for (b) the neighbors-based prediction, for an example patient: a 55-year-old male with BMI of $30 \mathrm{~kg} / \mathrm{m}^{2}$ and preoperative TUG time of $8 \mathrm{~s}$.

Performance via internal and external validation. Once the number of matches was fixed via tuning procedures in the training dataset, the within-sample and out-of-sample calibration was examined. The training dataset supplied donor data for both of these analyses. This mimics how the development and testing of the approach would work in practice. Model calibration was good, with close agreement between predicted and observed values of post-operative TUG times (Fig. 4).

\section{Discussion}

We developed and tested a novel, neighbors-based prediction for physical function following TKA. Via predictive mean matching, Body Mass Index (BMI), sex, age, and preoperative TUG time were used to identify the matches for an index patient. In our approach, the observed data from these matches were then used to generate a prediction for a new patient's TUG prognosis. One of our primary findings was the number of matches $(\mathrm{m}=35)$ required to generate predictions with optimal bias, coverage, and precision. This solution demonstrated very low bias and accurate coverage. Additionally, the $50 \%$ prediction interval was $2.33 \mathrm{~s}$, on average. This amounts to a $23 \%$ improvement in precision, compared to prognostic estimates derived from the whole sample (50\% prediction interval $=3.03 \mathrm{~s}$ ).

The predictions were well-calibrated in both the training and test datasets. In a temporally distinct test sample of patients with later surgical dates, the predictions performed accurately across all deciles of observed data. This was especially encouraging given the differences in patient characteristics between training and test datasets (Table 1). Moreover, national-level changes to TKA care and reimbursement occurred during the period of data collection $^{28}$. Such factors are likely to make external validation more challenging, but our initial analysis suggests the neighbor's based prediction approach is at least somewhat robust. To our knowledge, this is the first study to successfully validate a prediction model for physical function in TKA. 

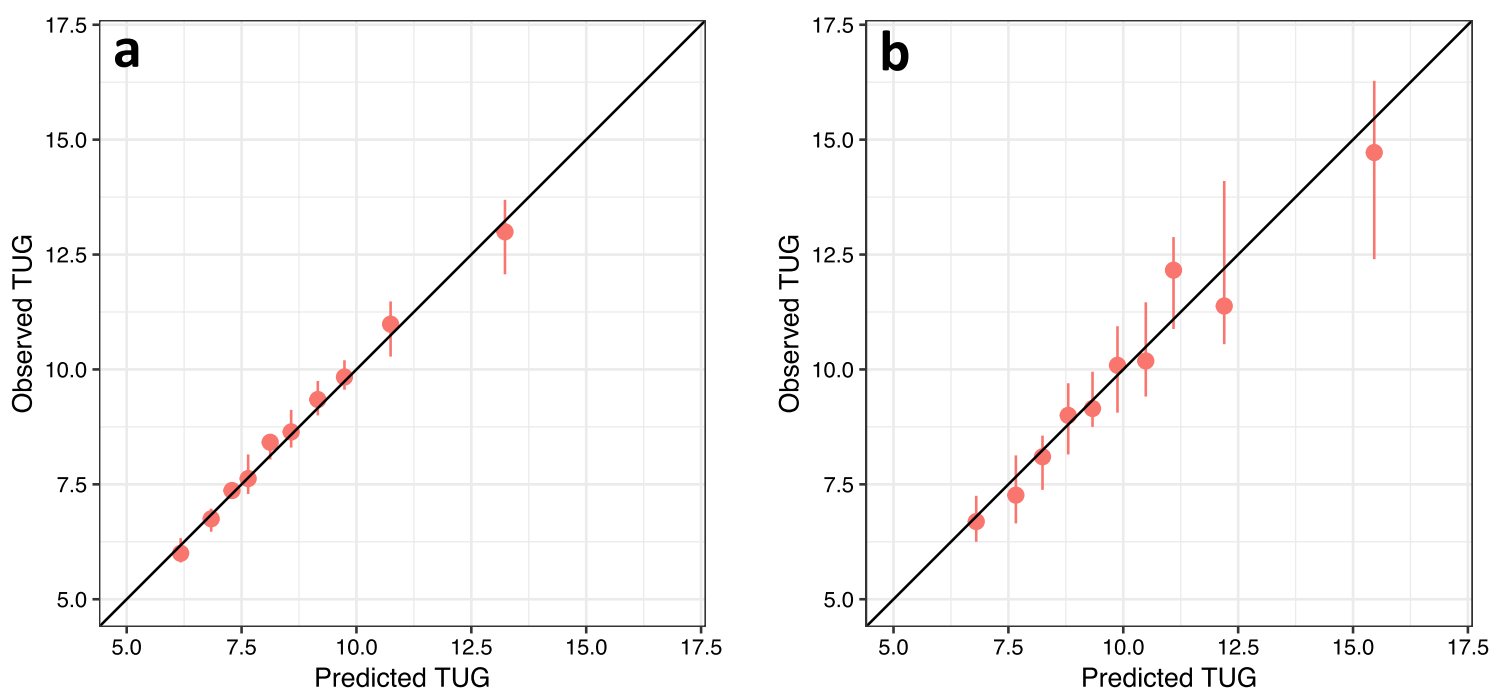

Figure 4. Calibration plots for neighbors-based predictions in: (a) training and (b) test datasets. Training and test datasets were divided into deciles according to the predicted TUG times. For each decile, the median observed TUG time is plotted against the median predicted TUG time. Error bars indicate the standard error of the median.

There are several features of the approach that may have contributed to the observed accuracy of the predictions. First, estimates were based on flexible models of empirical observations, which may have allowed for more realistic representations of recovery compared to previous approaches. Second, the selection of neighbors and subsequent prediction were performed independently for each patient. Thus, each patient served as the nucleus of his or her own prediction. This may have improved external validity since each individual patient's prediction was generated from similar patients' observed recovery. Finally, matches were determined by an adaptation of predictive mean matching around estimated 90-day TUG times. Therefore, the matching characteristics were each weighted according to the strength of the relation to the outcome of interest. This differs from more conventional sequential $k$-nearest neighbors' approaches, where the measure to express distances between patients is pre-set without an explicit role for the outcome of interest.

Our analysis was limited to the matching characteristics available in our source data (i.e., age, sex, BMI, and preoperative TUG time). The use of additional matching characteristics might allow for a further-refined matching strategy, resulting in improvements to the precision of the predictions. For example, patients' pain status, comorbidity status, or surgical variables (i.e., implant type, procedure type) might be expected to influence prognosis. Future analyses that incorporate these variables would be worth pursuing. However, it is likely that some unmeasured variables are co-linear with age, sex, BMI, and preoperative TUG time and are thus somewhat baked into the current analysis. Moreover, our results suggest that the neighbors-based predictions performed well even with a small number of matching characteristics.

A limitation of our study is the use of patient data from a small number of research and clinical datasets, as care paradigms and patient demographics may differ across settings and geographical locations. Additionally, our relatively high rates of missingness may be attributed to the challenges of performing rigorous data collection in the context of routine clinical practice. Thus, our study sample is likely to differ from other specific patient samples. Our calibration findings in a temporal validation are encouraging. Nevertheless, the prediction approach should be tested in prospectively enrolled participants to further examine the generalizability.

In conclusion, a novel neighbors-based prediction approach was used to estimate postoperative TUG times following TKA surgery, utilizing patient age, sex, BMI, and preoperative TUG time. Predictions performed accurately in estimating observed TUG times at any point during first six months following surgery, according to both within-sample and out-of-sample testing. This approach could be used to inform the understanding of functional prognosis for individual patients for this common elective surgery.

Received: 5 March 2020; Accepted: 5 July 2021

Published online: 18 August 2021

\section{References}

1. Bernstein, J. \& Derman, P. Dramatic increase in total knee replacement utilization rates cannot be fully explained by a disproportionate increase among younger patients. Orthopedics 37, e656-659. https://doi.org/10.3928/01477447-20140626-58 (2014).

2. Cheuy, V. A. et al. Influence of diabetes mellitus on the recovery trajectories of function, strength, and self-report measures after total knee arthroplasty. Arthritis Care Res. (Hoboken) https://doi.org/10.1002/acr.23741 (2018).

3. Bade, M. J., Kohrt, W. M. \& Stevens-Lapsley, J. E. Outcomes before and after total knee arthroplasty compared to healthy adults. J. Orthop. Sports Phys. Ther. 40, 559-567. https://doi.org/10.2519/jospt.2010.3317 (2010).

4. Judd, D. L., Eckhoff, D. G. \& Stevens-Lapsley, J. E. Muscle strength loss in the lower limb after total knee arthroplasty. Am. J. Phys. Med. Rehabil. 91, 220-226. https://doi.org/10.1097/PHM.0b013e3182411e49 (2012). 
5. Weiss, J. M. et al. What functional activities are important to patients with knee replacements? Clin. Orthop. Relat. Res., 172-188 (2002).

6. Alemi, F., Erdman, H., Griva, I. \& Evans, C. H. Improved statistical methods are needed to advance personalized medicine. Open Transl. Med. J. 1, 16-20. https://doi.org/10.2174/1876399500901010016 (2009).

7. Ekman, I. et al. Person-centered care-ready for prime time. Eur. J. Cardiovasc. Nurs. 10, 248-251. https://doi.org/10.1016/j.ejcnu rse.2011.06.008 (2011)

8. Leplege, A. et al. Person-centredness: conceptual and historical perspectives. Disabil. Rehabil. 29, 1555-1565. https://doi.org/10. 1080/09638280701618661 (2007).

9. Kittelson, A. J., Hoogeboom, T. J., Schenkman, M., Stevens-Lapsley, J. E. \& van Meeteren, N. L. U. Person-centered care and physical therapy: a "people-like-me" approach. Phys. Ther. 100, 99-106. https://doi.org/10.1093/ptj/pzz139 (2020).

10. Dawes, M. et al. Sicily statement on evidence-based practice. BMC Med. Educ. 5, 1. https://doi.org/10.1186/1472-6920-5-1 (2005).

11. Sanchez-Santos, M. T. et al. Development and validation of a clinical prediction model for patient-reported pain and function after primary total knee replacement surgery. Sci. Rep. 8, 3381. https://doi.org/10.1038/s41598-018-21714-1 (2018).

12. Kennedy, D. M., Stratford, P. W., Riddle, D. L., Hanna, S. E. \& Gollish, J. D. Assessing recovery and establishing prognosis following total knee arthroplasty. Phys. Ther. 88, 22-32. https://doi.org/10.2522/ptj.20070051 (2008).

13. Mizner, R. L., Petterson, S. C., Stevens, J. E., Axe, M. J. \& Snyder-Mackler, L. Preoperative quadriceps strength predicts functional ability one year after total knee arthroplasty. J. Rheumatol. 32, 1533-1539 (2005).

14. Chen, G.H. \& Shah, D., Explaining the success of nearest neighbor methods in prediction. Found. Trends Mach. Learn. 10, 337-588. https://doi.org/10.1561/2200000064 (2018).

15. Page, M. G. et al. Distinguishing problematic from nonproblematic postsurgical pain: a pain trajectory analysis after total knee arthroplasty. Pain 156, 460-468. https://doi.org/10.1097/01.j.pain.0000460327.10515.2d (2015).

16. van Buuren, S. Curve matching: a data-driven technique to improve individual prediction of childhood growth. Ann. Nutr. Metab. 65, 227-233. https://doi.org/10.1159/000365398 (2014).

17. Stevens-Lapsley, J. E., Bade, M. J., Shulman, B. C., Kohrt, W. M. \& Dayton, M. R. Minimally invasive total knee arthroplasty improves early knee strength but not functional performance: a randomized controlled trial. J. Arthroplasty https://doi.org/10. 1016/j.arth.2012.02.016 (2012).

18. Stevens-Lapsley, J. E., Balter, J. E., Wolfe, P., Eckhoff, D. G. \& Kohrt, W. M. Early neuromuscular electrical stimulation to improve quadriceps muscle strength after total knee arthroplasty: a randomized controlled trial. Phys. Ther. 92, 210-226. https://doi.org/ 10.2522/ptj.20110124 (2012).

19. Bade, M. J. et al. Early high-intensity versus low-intensity rehabilitation after total knee arthroplasty: a randomized controlled trial. Arthritis Care Res. (Hoboken) 69, 1360-1368. https://doi.org/10.1002/acr.23139 (2017).

20. Loyd, B. J., Kittelson, A. J., Forster, J., Stackhouse, S. \& Stevens-Lapsley, J. Development of a reference chart to monitor postoperative swelling following total knee arthroplasty. Disabil. Rehabil. 42, 1767-1774. https://doi.org/10.1080/09638288.2018.1534005 (2020).

21. Naylor, J. M. et al. Minimal detectable change for mobility and patient-reported tools in people with osteoarthritis awaiting arthroplasty. BMC Musculoskelet. Disord. 15, 235. https://doi.org/10.1186/1471-2474-15-235 (2014).

22. Podsiadlo, D. \& Richardson, S. The timed "Up \& Go": a test of basic functional mobility for frail elderly persons. J. Am. Geriatr. Soc. 39, 142-148. https://doi.org/10.1111/j.1532-5415.1991.tb01616.x (1991).

23. De Kroon, M. L., Renders, C. M., Van Wouwe, J. P., Van Buuren, S. \& Hirasing, R. A. The Terneuzen birth cohort: BMI changes between 2 and 6 years correlate strongest with adult overweight. PLoS ONE 5, e9155. https://doi.org/10.1371/journal.pone.00091 55 (2010).

24. Anderson, C., Hafen, R., Sofrygin, O. \& Ryan, L. Comparing predictive abilities of longitudinal child growth models. Stat. Med. 38, 3555-3570. https://doi.org/10.1002/sim.7693 (2019).

25. van Buuren, S. Broken stick model for irregular longitudinal data. J. Stat. Softw. (in review).

26. van Buuren, S. Flexible imputation of missing data. Second edition. Chapman and Hall/CRC Press, Taylor \& Francis Group (2018).

27. Rigby, R. A.\& Stasinopoulos, D. M. Generalized Additive Models for Location, Scale and Shape. Journal of the Royal Statistical Society. Series C (Applied Statistics) 54,507-554 https://doi.org/10.1111/j.1467-9876.2005.00510.x (2005).

28. Medicare Program; Comprehensive care for joint replacement payment model for acute care hospitals furnishing lower extremity joint replacement services. Department of Health and Human Services, Center for Medicare and Medicaid Services. Federal Registry. 80, 73273-73554 (2015).

\section{Author contributions}

All authors contributed to the study conception and design. CK, SvB, KC, and AK contributed to the data analysis and initial drafting of the manuscript. All authors contributed to revisions of the manuscript and approved of the final version.

\section{Funding}

This study was funded by grants through the Agency for Healthcare Research and Quality (R03 HS024316, R01 HS025692) and National Institutes of Health (K12 HD055931).

\section{Competing interests}

The authors declare no competing interests.

\section{Additional information}

Supplementary Information The online version contains supplementary material available at https://doi.org/ 10.1038/s41598-021-94838-6.

Correspondence and requests for materials should be addressed to A.J.K.

Reprints and permissions information is available at www.nature.com/reprints.

Publisher's note Springer Nature remains neutral with regard to jurisdictional claims in published maps and institutional affiliations. 
(c) (i) Open Access This article is licensed under a Creative Commons Attribution 4.0 International cc) License, which permits use, sharing, adaptation, distribution and reproduction in any medium or format, as long as you give appropriate credit to the original author(s) and the source, provide a link to the Creative Commons licence, and indicate if changes were made. The images or other third party material in this article are included in the article's Creative Commons licence, unless indicated otherwise in a credit line to the material. If material is not included in the article's Creative Commons licence and your intended use is not permitted by statutory regulation or exceeds the permitted use, you will need to obtain permission directly from the copyright holder. To view a copy of this licence, visit http://creativecommons.org/licenses/by/4.0/.

(C) The Author(s) 2021 